\title{
Measuring and Optimizing the Momentum Aperture in a Particle Accelerator
}

\author{
C. Steier, ${ }^{1}$ D. Robin, ${ }^{1}$ L. Nadolski, ${ }^{1,2}$ W. Decking, ${ }^{3}$ Y. Wu, ${ }^{1,4}$ and J. Laskar ${ }^{2}$ \\ ${ }^{1}$ Lawrence Berkeley National Laboratory, Berkeley, CA 94720, USA \\ ${ }^{2}$ Astronomie et Systèmes Dynamiques, IMC-CNRS, 75014 Paris, France \\ ${ }^{3}$ DESY, 22603 Hamburg, Germany \\ ${ }^{4}$ Duke University, Durham, NC 27708, USA
}

(Dated: February 18, 2002)

\begin{abstract}
Particle motion in storage rings is confined by various aperture limits, the size of which restricts the performance of the ring in terms of injection efficiency, lifetime, etc. Intra-beam scattering makes particles sweep a large portion of the phase space where their motion may eventually be resonantly or chaotically excited to large amplitudes leading to collision with the vacuum chamber. We report here the studies performed at the Advanced Light Source (ALS) on the on- and off-momentum particle motion that provides a good understanding of these limitations. Using off-momentum simulations and experiments together with Frequency Map Analysis, we could precisely correlate beam loss areas with resonance locations. The very good agreement between simulations and experiments allowed us to provide guidance for avoiding these dangerous areas. This analysis results in predictive improvements of the momentum aperture which actually led to a lifetime increase of $25 \%$ at the ALS for very high bunch charge.
\end{abstract}

\section{INTRODUCTION}

Charged particle storage rings are used for a variety of science and technology applications - for example as synchrotron radiation light sources for biology, chemistry, and materials science, and as colliders for highenergy physics. In these storage rings, bunched particle beams travelling near the speed of light circulate for many hours. The motion of an individual particle can be described in terms of transverse (betatron) and longitudinal (synchrotron) motions with respect to the reference particle. Some of these particles may be lost due to various aperture limitations. The momentum aperture is defined as the maximum momentum deviation that a particle can have without becoming unstable and being lost by colliding with the vacuum chamber of the storage ring. The momentum aperture is determined by the complex 6-dimensional dynamics of the particle. Because of the complexity of the particle dynamics, up to now there have been unexplained discrepancies between the predicted and measured momentum aperture [1-11].

Having a good understanding of the momentum aperture is important because it is one of the main performance limitations for storage rings. A primary reason that particles are lost is that they gain or lose enough momenta to exceed the momentum aperture via scattering with gas particles in the ring (Bremsstrahlung [12]), via collisions with other particles within the bunch (intra-beam and Touschek scattering [13]), or via collisions with particles in other bunches (beam-beam Bremsstrahlung [14]). The rate at which particles are lost determines the beam lifetime. Long beam lifetimes are desirable to the users of synchrotron light source rings and collider rings. At synchrotron light facilities, long lifetimes increase the integrated photon flux, reduce the frequency of refills, and improve the stability of the beam by reducing thermal loading effects due to the varying current. For collider rings long beam lifetimes are also important because they increase the integrated luminosity delivered by the collider.

In those storage rings where the dominant lifetime limitation process is Touschek scattering, the lifetime strongly depends on the momentum aperture - stronger than a quadratic dependency. Therefore, it is desirable to operate such storage rings with as large a momentum aperture as possible. This is particularly true for the third generation synchrotron light source rings in which circulating electron/positron bunches have a large charge density, resulting in intense Touschek scattering. The Advanced Light Source (ALS) at Lawrence Berkeley National Laboratory (LBNL) is such a third generation light source operated for users since 1993 [1]. The nominal operation parameters of the ALS are shown in Table I. As seen in the table the Touschek lifetime of 9 hours is much shorter than the vacuum lifetime of 60 hours. Consequently, the Touschek lifetime limited ALS would benefit greatly from a larger momentum aperture.

In fact the measured momentum aperture at the ALS as well as other existing light sources and colliders are smaller than the predicted values (for third generation light sources the predicted values range between $3 \%$ and $5 \%[1,3,7]$ whereas measured values range between $1 \%$ and $3 \%[4-8,10,15])$. Because lifetime is such an important performance parameter, one of the main design goals of new and future light sources such as SLS and SOLEIL [16, 17] is to achieve even larger momentum apertures - larger than $5 \%$. Therefore it is important to understand what limits the momentum aperture in existing storage rings. This knowledge will help improve the performance of existing light sources as well as to help accurately predict and optimize the performance of future storage rings.

The particle dynamics and momentum aperture have been extensively studied at the ALS. Simple yet powerful measurement techniques have been used to gain insight into the limitation of the momentum aperture. The mea- 
TABLE I: Nominal ALS parameters.

\begin{tabular}{|c|l|c|}
\hline Parameter & Description & \\
\hline$E_{0}$ & Beam energy & $1.5-1.9 \mathrm{GeV}$ \\
$C$ & Circumference & $196.8 \mathrm{~m}$ \\
$\nu_{x}$ & Horizontal tune & 14.25 \\
$\nu_{y}$ & Vertical tune & 8.20 \\
$\xi_{x}^{\text {nat }}$ & Horizontal natural chromaticity & -24.6 \\
$\xi_{y}^{\text {nat }}$ & Vertical natural chromaticity & -26.7 \\
$\varepsilon_{x}$ & Horizontal emittance & $3.5-5.5 \mathrm{~nm} \mathrm{rad}$ \\
$\kappa=\epsilon_{y} / \epsilon_{x}$ & Typical emittance ratio & $0.5-7 \%$ \\
$I_{b}$ & Bunch current $(400 \mathrm{~mA}$ multibunch fill) & $\approx 1.5 \mathrm{~mA}$ \\
\hline \multicolumn{2}{|l}{} & \\
\hline$\tau_{\text {vac }}$ & Lifetimes (400 mA, $1.9 \mathrm{GeV})$ & $\approx 60 \mathrm{~h}$ \\
$\tau_{\text {tous }}$ & Vacuum lifetime & $\approx 9 \mathrm{~h}$ \\
$\tau_{\text {total }}$ & Touschek lifetime $(3.5 \%$ coupling) & $\approx 8 \mathrm{~h}$ \\
\hline
\end{tabular}

surements clearly show that the major limitation to the momentum aperture is the transverse beam dynamics, causing Touschek scattered particles to eventually reach large vertical amplitudes where they are lost on the vacuum chamber. Analysis of the measurement data using Frequency Map Analysis allows us to understand the details of the beam loss - identifying those resonances that limit the momentum aperture. The knowledge gained as a result of these measurements allows us to adjust the machine parameters to improve the lifetime.

In this paper the results of these studies are presented. In Section II we discuss the Touschek scattering and the various apertures (RF, Physical, and Dynamic) that limit the momentum aperture. In Section III measurements of the momentum aperture are presented. Through these measurements we show that the dynamic aperture is the dominant effect that limits the momentum aperture and that this limitation strongly depends upon the machine conditions. In Section IV we examine the mechanism of Touschek scattering in more detail — illustrating how Touschek scattered particles can ultimately reach large vertical amplitudes through nonlinear resonance excitation and diffusion, causing them to collide with the vacuum chamber. In this section we also discuss using the off-momentum transverse dynamics and Frequency Map Analysis to gain insight into the full 6-dimensional dynamics. In Section $\mathrm{V}$ measurements of the off-momentum dynamics are presented. A comparison is made between the off-momentum dynamics and the momentum apertures presented in Section III. In Section VI we present simulations of the off-momentum dynamics using our model of the ALS and comparison is made with the measurements (shown in Section V). Section VII shows the relation between the momentum aperture and the vertical physical aperture, while we end with some concluding remarks in Section VIII.

\section{TOUSCHEK LIFETIME AND MOMENTUM APERTURE}

In this section we will first summarize the various aperture limitations and then discuss one of the processes (Touschek scattering) that leads to particle amplitudes which may exceed these aperture limitations.

In a storage ring, particles perform oscillations around a central periodic closed orbit. The 6 -dimensional phase space coordinates are $\left(x, p_{x}, y, p_{y}, \delta, l\right)$, where $x$ and $y$ are the horizontal and vertical position offsets, $p_{x}$ and $p_{y}$ are the normalized horizontal and vertical momenta, $\delta$ is the relative momentum deviation, and $l$ is the relative path length with respect to the synchronous particle in the longitudinal direction. The particle oscillation is guided by magnetic fields for the transverse motion and electric fields (radiofrequency $-\mathrm{RF}-$ ) for the longitudinal motion. The final boundary for particle motion is the vacuum chamber wall. Every particle will eventually be lost at the vacuum chamber. Depending on the amplitude and orientation of the oscillation we distinguish three aperture limitations in the storage ring:

- The RF-momentum aperture, $\varepsilon_{\mathrm{RF}}$, which corresponds to the maximum particle momentum deviation at which the longitudinal motion remains stable due to the confinement of the RF-fields.

- The physical aperture, $x_{\text {phys }}$, which is related to the linear motion. The transverse magnetic guide fields are set up in a way to ensure stable particle motion. At low amplitudes the motion can be described by linear optical functions. The linear motion amplitude which leads to a particle loss on the vacuum chamber is called the physical aperture.

- The dynamic aperture, $x_{\mathrm{dyn}}$, which is related to the nonlinear motion. With increasing amplitude the 
motion of the particle becomes more and more nonlinear. The dynamic aperture can be defined as the smallest initial amplitude of the particle whose motion will cause its amplitude to increase resonantly or diffusively until the particle is lost against the vacuum chamber $[7,16,18,19]$.

In the next three subsections these apertures will be discussed in more detail which will be followed in a fourth subsection by a discussion of the Touschek scattering process.

\section{A. RF-momentum Aperture}

If the initial relative momentum deviation, $\delta_{0}$, of a particle is larger in absolute value than the RF-momentum aperture, $\varepsilon_{\mathrm{RF}}$, the particle stays outside of the stable area in the longitudinal phase space. Its momentum will continue either to increase or decrease until it reaches a sufficiently large value that the particle is lost from the ring. The size of the RF-momentum aperture (or RF-bucket height) provided by the accelerating voltage, $V_{\mathrm{RF}}$, in the cavity is [20]:

$$
\varepsilon_{\mathrm{RF}}= \pm\left(\frac{2 U_{0}}{\pi \alpha_{c} h E_{0}} \sqrt{\left(\frac{V_{\mathrm{RF}}}{U_{0}}\right)^{2}-1}-\arccos \left(\frac{U_{0}}{V_{\mathrm{RF}}}\right)\right)^{\frac{1}{2}}
$$

where $\alpha_{c}$ is the momentum compaction factor, $U_{0}$ is the radiation loss per turn, $h$ is the harmonic number, and $E_{0}$ is the nominal energy. Equation 1 is derived neglecting the details of quantum excitation and radiation damping, which is valid for most storage rings. It should be mentioned that in the presence of radiation damping, not all particles scattered outside the RF-momentum aperture will be lost from the ring. It has been observed [21] that there is a finite probability that some of the particles may eventually be captured in other buckets. However in most cases the fraction of particles recaptured is a small portion of those scattered outside the RF-momentum aperture.

\section{B. Physical Aperture}

Assuming linear particle motion, the largest amplitude a particle can have for a given momentum deviation $\delta$ without hitting the vacuum chamber is determined by the smallest vacuum chamber size normalized by the $\beta$ function in the ring,

$$
x_{\mathrm{phys}}(\delta, s)=\sqrt{\min _{s_{1} \in[0, L]}\left[\frac{\left(x_{v c}^{ \pm}\left(s_{1}\right)-\eta_{x}\left(s_{1}\right) \delta\right)^{2}}{\beta_{x}\left(s_{1}\right)}\right] \beta_{x}(s)},
$$

with $\eta_{x}, \beta_{x}$ being the dispersion and $\beta$-function, $x_{v c}^{ \pm}$the vacuum chamber half-size, and $s$ the position along the ring of circumference $L$. This is termed the physical aperture. In a perfect storage ring with mid-plane symmetry, the vertical dispersion is zero. Therefore, the vertical physical aperture, $y_{\text {phys }}$, calculated using Equation 2 by replacing $x$ by $y$, is momentum independent.

\section{Dynamic Aperture}

At large amplitudes, the motion of a particle is nonlinear and at sufficiently large amplitudes the motion can be resonantly excited or chaotic. Unlike the physical aperture, the dynamic aperture separating stable (within a given number of turns) and unstable trajectories is not a hard boundary. Rather there exist high diffusion zones where the particle can diffuse to large transverse amplitudes. The presence of the vacuum chamber adds another feature: the motion of some particles that are resonantly excited to larger amplitudes can be dynamically stable but the particles can still be lost because of the finite size of a smaller vacuum chamber. The size of the dynamic aperture thus depends also, in this definition, on the size of the vacuum chamber.

\section{Touschek Scattering}

Several mechanisms exist to excite large amplitude oscillations. During injection particles enter the ring far away from the closed orbit and thus perform large amplitude oscillations. For example at the ALS a typical injection offset is about $10 \mathrm{~mm}$ which is enough to clear the injection septum. Particles may also scatter with the residual gas and start oscillations. In densely filled bunches particles may scatter among themselves (Touschek effect). This process has been studied extensively at the ALS leading to a better understanding about how the different apertures contribute to the momentum aperture and ultimately the lifetime.

A particle located in the center of an electron bunch is Touschek scattered to some momentum deviation, $\delta_{0}$. So with respect to the nominal particle, the coordinates of this particle are $\left(x=0, p_{x}=0, y=0, p_{y}=0, \delta=\right.$ $\left.\delta_{0}, l=0\right)$ - in the local frame of reference the particle may be scattered to any angle but in the laboratory frame it is mostly longitudinal. After this momentum change, the particle starts an oscillation around a new closed orbit [20]. With the assumption that the dispersion function exists only in the horizontal plane, the particle coordinates with respect to this orbit are: $\left(x=-\eta_{x} \delta_{0}, p_{x}=-\eta_{x}^{\prime} \delta_{0}, y=0, p_{y}=0, \delta=\delta_{0}, l=0\right)$. Assuming linear motion the induced betatron amplitude of this oscillation can be derived as:

$$
x_{\text {ind }}(s, \delta)=\sqrt{\beta_{x}(s) H_{x}(s)} \delta \text {, }
$$


with

$$
H_{x}(s)=\gamma_{x}(s) \eta_{x}(s)^{2}+2 \alpha_{x}(s) \eta_{x}(s) \eta_{x}^{\prime}(s)+\beta_{x}(s) \eta_{x}^{\prime}(s)^{2}
$$

where $\alpha_{x}, \beta_{x}$, and $\gamma_{x}$ are the Twiss functions [22].

Equations 2, 3 and 4 are not valid for large momentum deviations and transverse amplitudes [23]. Strong sextupoles lead to higher order dispersion which alters the off-momentum closed orbit and Twiss functions. However at the ALS the difference between the momentum independent and momentum dependent calculations of the optical functions and the closed orbit is less than $5 \%$ for momentum deviations of up to $5 \%$.

The transverse momentum aperture, $\varepsilon_{\text {trans }}(s)$, defines the maximum momentum deviation a particle can get at a Touschek scattering event without being lost on any of the transverse aperture limitations. At a given position $s, \varepsilon_{\text {trans }}(s)$ can be calculated by solving the following implicit equation:

$\sqrt{\beta_{x}(s) H_{x}(s)} \varepsilon_{\mathrm{trans}}=\min \left[x_{\mathrm{phys}}\left(\varepsilon_{\mathrm{trans}}, s\right), x_{\mathrm{dyn}}\left(\varepsilon_{\mathrm{trans}}, s\right)\right]$,

where $x_{\text {dyn }}$ is computed with respect to the offmomentum closed orbit. For Touschek scattered particles the momentum deviation, $\delta$, can be either positive or negative. Therefore the dynamic aperture limitation given in equation 5 can be either in the positive or negative $x$ direction.

At any position, $s$, of the ring the absolute momentum aperture is the smaller of the s-independent RFmomentum aperture, $\varepsilon_{\mathrm{RF}}$, and the s-dependent transverse momentum aperture, $\varepsilon_{\text {trans }}(s)$.

As an example Figure 1 illustrates a comparison of the maximum allowable apertures and the induced oscillation amplitudes (thin dashed lines). In the straight section, where there is no dispersion, the induced amplitude is zero. This means the particle will only change its energy but not start a betatron oscillation. In the arc section with a finite dispersion the induced amplitude shows a linear behavior as can be seen from Equation 3. The induced amplitude is (nearly) constant in the arcs because $H_{x}$ is (nearly) constant in the arcs.

The thick lines represent the various apertures. The vertical solid lines show the RF-momentum aperture with $\varepsilon_{\mathrm{RF}}=3 \%$, corresponding to the present available RFvoltage (for $1.5 \mathrm{GeV}$ ). The dashed-dotted line shows the physical apertures $x_{\text {phys }}$. The solid curved line is a sketch of the dynamic aperture. As discussed later, the size and the shape of the dynamic aperture strongly depends upon the machine conditions (i.e. tunes, chromaticities, coupling).

The momentum aperture is defined as the smallest crossing point of the induced amplitude and the smallest of the apertures. As the induced amplitude varies around the ring so does the momentum aperture. Figure 2 shows the momentum aperture for one cell of the ALS. The solid line is the momentum aperture due to

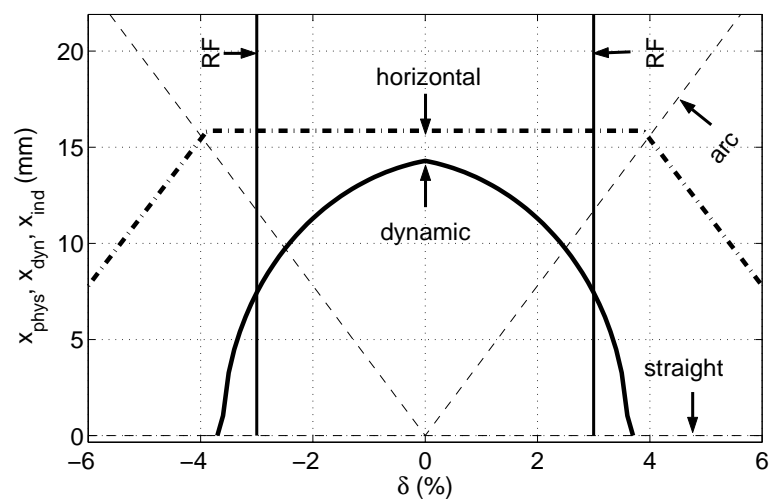

FIG. 1: Contributions of the different apertures to the total momentum aperture as a function of the relative momentum deviation $\delta$. The thin dashed lines represent the induced amplitudes, $x_{\text {ind }}$, for an arc or a straight section of the ring. Dashed-dotted lines stand for the physical apertures, $x_{\mathrm{phys}}$, the solid line is the dynamic aperture, $x_{\mathrm{dyn}}$, and the vertical lines show the RF-momentum aperture, $\varepsilon_{\mathrm{RF}}$. This figure as well as the following ones is plotted at the injection point $\left(\beta_{x}\left(s_{0}\right)=12 \mathrm{~m}\right)$.

transverse limitations as derived from Figure 1 (transverse momentum aperture). The dashed-dotted line is the RF-momentum aperture. In this case the momentum aperture, $\varepsilon(s)$, is defined by the RF-momentum aperture, $\varepsilon_{\mathrm{RF}}$, in the straight section and by the transverse momentum aperture, $\varepsilon_{\text {trans }}$, in the arcs.

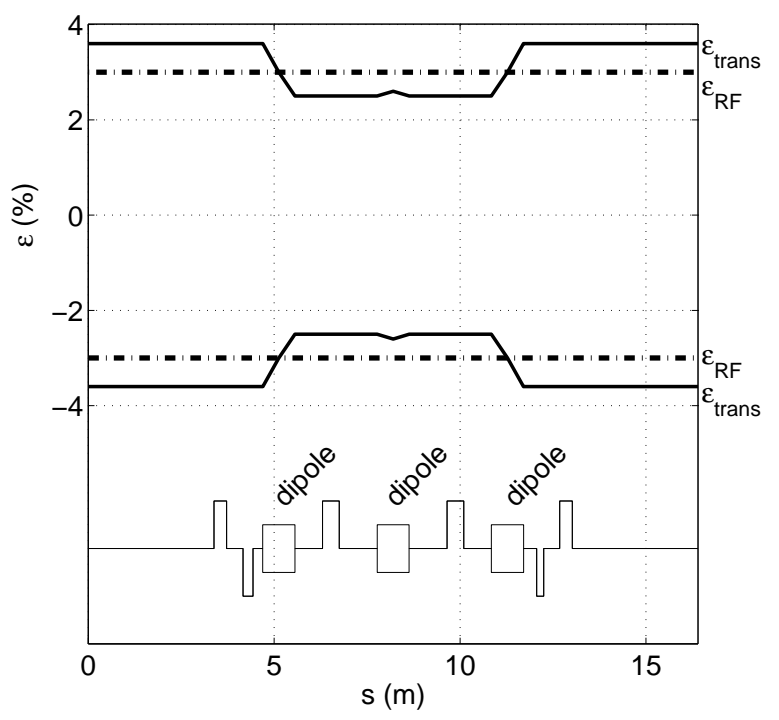

FIG. 2: Momentum aperture, $\varepsilon$, along one of the 12 cells of the ALS storage ring. The solid line is the momentum aperture from transverse limitations as derived from Figure 1 and the dashed-dotted line is the RF-momentum aperture. The cell layout of the ALS is shown in the lower part of the figure with the three dipoles of the achromat separated by quadrupole magnets.

Having defined the momentum aperture one can now 
calculate the Touschek lifetime. Assuming a flat beam, and thus the main contribution of the velocity spread coming from horizontal motion the lifetime due to the Touschek effects is given by [13]:

$$
\tau_{\text {tou }}=\frac{E_{0}^{3}}{L I_{b}} \int \frac{\varepsilon(s)^{2} V_{b}(s) \sigma_{x}^{\prime}(s)}{\ln \left(\frac{1}{1.78} \frac{\sigma_{x}^{\prime}(s)^{2} E_{0}^{2}}{\varepsilon(s)^{2}}\right)} d s,
$$

where $L$ is the length of the ring, $I_{b}$ is the bunch current, $E_{0}$ is the beam energy, $V_{b}(s)=\sigma_{L} \sigma_{x}(s) \sigma_{y}(s)$ is the bunch volume, and $\sigma_{x}^{\prime}(s)$ is the angular spread of electrons in the bunch. Note the bunch volume, angular spread and momentum aperture vary around the ring.

\section{MEASUREMENTS OF THE MOMENTUM APERTURE}

An easy way to study whether the momentum aperture of a ring is solely determined by the RF-momentum aperture, or whether it is also limited by other apertures, is to vary the RF-voltage and measure the lifetime. At the ALS, measurements of the Touschek lifetime as a function of the RF-voltage presented here were conducted at a beam energy of $1.5 \mathrm{GeV}$ under different operating conditions. At each measurement point the synchrotron tune, $\nu_{s}$, was measured simultaneously, allowing one to calculate the bunch length, $\sigma_{L}$, and the RF-voltage $V_{\mathrm{RF}}[22]$ :

$$
\begin{gathered}
\sigma_{L}=\frac{\alpha_{c} \sigma_{E} L}{2 \pi \nu_{s}}, \\
V_{\mathrm{RF}}=\frac{1}{e} \sqrt{\frac{\nu_{s}^{4}(2 \pi)^{2} E_{0}^{2}}{\left(\alpha_{c} h\right)^{2}}+U_{0}^{2}},
\end{gathered}
$$

where $\sigma_{E}$ is the energy spread of the beam, $U_{0}$ the energy loss per turn due to synchrotron radiation and $e$ the charge of an electron. Knowing $V_{\mathrm{RF}}$, it is possible to calculate the RF-momentum aperture (see Equation 1). To enhance the effect of the Touschek scattering over other lifetime effects, a high current per bunch was filled in a few equally spaced bunches. The low number of bunches avoids multi-bunch instabilities. The beam conditions thus were $1.5 \mathrm{~mA} /$ bunch and a train of 16 bunches (out of 328) filled.

In Equation 6, the bunch volume is adjusted according to the changing RF-voltage $\left(V_{b} \propto \sqrt{1 / V_{\mathrm{RF}}}\right)$, and is corrected by a constant factor, $A$, which takes into account any volume changes like variation of the coupling, instabilities, etc. There is also assumed to be only 2 transverse momentum apertures, $\varepsilon_{\text {trans }}(s)$, - one for the straights, $\varepsilon_{\text {straight }}$, and one for the arcs, $\varepsilon_{\text {arc }}$ (see Figure 2 ). With these simplifications, Equation 6 becomes:

$$
\tau_{\text {tous }}=\frac{A E_{0}^{3} \sigma_{L}\left(\nu_{s}\right)}{L I_{b}}\left[\varepsilon_{\operatorname{arc}}^{2} \int_{\operatorname{arc}} \frac{\sigma_{x}(s) \sigma_{y}(s) \sigma_{x}^{\prime}(s)}{\ln \left(\frac{1}{1.78} \frac{\sigma_{x}^{\prime}(s)^{2} E_{0}^{2}}{\varepsilon_{\text {arc }}^{2}}\right)} d s\right.
$$

$$
\left.+\varepsilon_{\text {straight }}^{2} \int_{\text {straight }} \frac{\sigma_{x}(s) \sigma_{y}(s) \sigma_{x}^{\prime}(s)}{\ln \left(\frac{1}{1.78} \frac{\sigma_{x}^{\prime}(s)^{2} E_{0}^{2}}{\varepsilon_{\text {straight }}^{2}}\right)} d s\right],
$$

and the data can be fitted by nonlinear least squares with just three parameters, $A, \varepsilon_{\text {straight }}$, and $\varepsilon_{\text {arc }}$.

As an example, Figure 3 shows the measurement data of lifetime versus RF-momentum aperture for the following storage ring conditions: tunes set to $\nu_{x}=14.25$ and $\nu_{y}=8.20$, chromaticities set to $\xi_{x}=0.4$ and $\xi_{y}=4.4$, and an emittance ratio of about $7 \%$. The solid line represents a fit of the data using all 3 parameters $A, \varepsilon_{\text {arc }}$, $\varepsilon_{\text {straight }}$, the dashed-dotted line shows the lifetime behavior if only the RF-momentum aperture determined the momentum aperture and the dashed line shows the lifetime behavior if only the RF-momentum aperture and the transverse momentum aperture in the arcs would determine the momentum aperture. The measurement data shows that for large RF-momentum apertures, the momentum apertures in both the straight sections and the arcs are primarily determined by the transverse momentum aperture. Fitting the data results in transverse momentum apertures of $\varepsilon_{\text {straight }}=2.6 \%$ and $\varepsilon_{\text {arc }}=1.75 \%$.

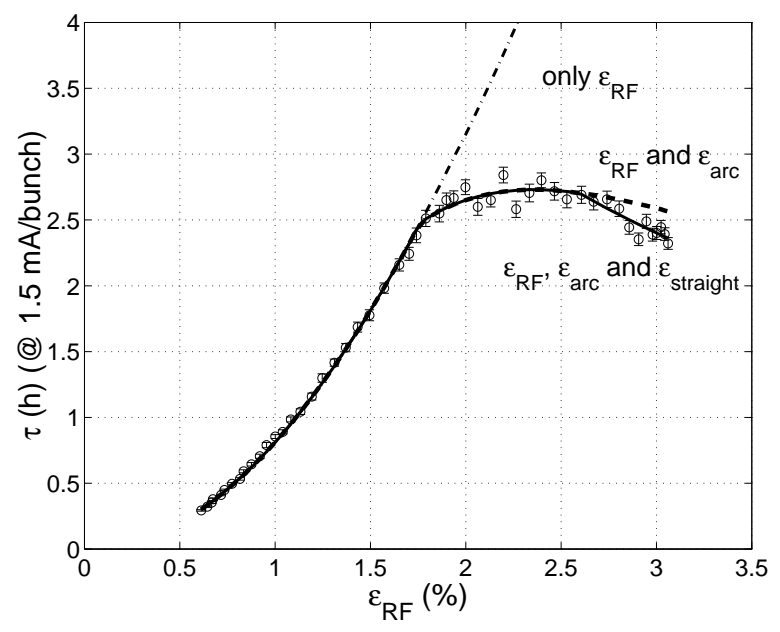

FIG. 3: Measurement of beam lifetime, $\tau$, as a function of the RF-momentum aperture with lines representing the fitted lifetime behavior (solid), the lifetime with no transverse aperture restrictions (dashed-dotted) and with transverse aperture restriction only for particles scattered in the arcs (dashed).

Figure 4 shows measurement data for three different sets of chromaticities, with the tunes at $\nu_{x}=14.25$ and $\nu_{y}=8.20$, and the coupling adjusted to about $7 \%$. As one can clearly see, the momentum aperture and thus the lifetime is very sensitive to the linear chromaticities. The maximum achievable lifetimes differ in these cases by more than a factor of 2 . It is best for the nominal chromaticities $\left(\xi_{x}=0.4, \xi_{y}=1.4\right)$, where the ALS is operated at in multi-bunch operation (solid line), and significantly worse (dashed-dotted line) for a vertical chromaticity increased by 3 units $\left(\xi_{x}=0.4, \xi_{y}=4.4\right)$. The third case (dashed line) is a case where both the hor- 
izontal and vertical chromaticities are increased $\left(\xi_{x}=\right.$ $\left.2.3, \xi_{y}=4.3\right)$ and the resulting maximum lifetime is $25 \%$ longer than in the case where just the vertical chromaticity is increased. At first sight this result might seem counter-intuitive. Yet the reasons leading to this choice will be fully evident in the next section where the particle momentum dynamics is explored in an extended way.

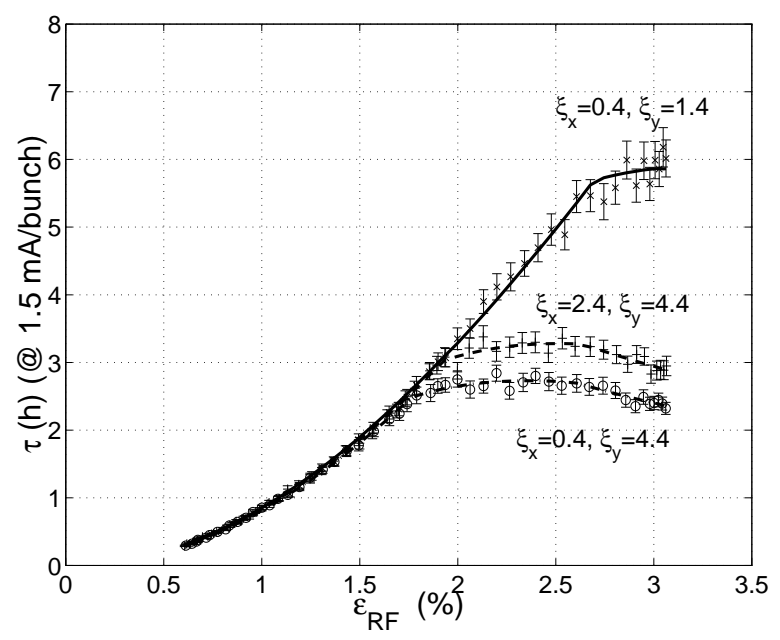

FIG. 4: Measurement of the beam lifetime, $\tau$, as a function of the RF-momentum aperture for three different sets of chromaticities. The lines represent the fitted lifetime behavior for the nominal chromaticity case (solid line), a case with higher vertical chromaticity (dashed-dotted line), and a case with higher chromaticities in both transverse planes (dashed line). The emittance coupling in all cases was about $7 \%$ and the betatron tunes were $\nu_{x}=14.25$ and $\nu_{y}=8.20$. One can see that the dynamic momentum aperture has a severe impact on the overall momentum aperture and therefore the Touschek lifetime.

The latter case is of special interest for machine operation: while operating the ring with large single bunch currents (so called two-bunch mode) the vertical chromaticity has to be significantly increased in order to damp single bunch instabilities. From Figure 4 it is clear that the dynamic aperture impacts the momentum aperture. This can be inferred from the fact that when the chromaticity is changed, the size of the physical aperture remains fixed whereas the size of the momentum aperture changes.

For all 3 cases shown in Figure 4 the fitted transverse apertures are listed in Table II. As shown in the table, the transverse apertures in the arcs vary by a large factor. Additional measurements were made for many other machine conditions: different values of coupling, chromaticities, tunes, vertical aperture and insertion device settings $[5,6]$.

It has thus been demonstrated that at the ALS, the dynamic aperture limits the momentum aperture. In sections $\mathrm{V}$ and VI we describe the measurements made to understand these limitations in more detail and to get a clear picture showing the dynamics of particle loss. These experiments are conducted for the three different
TABLE II: Fitted momentum apertures for three different conditions.

\begin{tabular}{|c|c|c|c|}
\hline $\begin{array}{c}\text { Chromaticity } \\
\text { (hor./vert.) }\end{array}$ & $\varepsilon_{\text {straight }}$ & $\varepsilon_{\text {arc }}$ & $\mathrm{A}$ \\
\hline$\xi_{x}=0.4, \xi_{y}=1.4$ & $>3 \%$ & $2.65 \pm 0.02 \%$ & $1.01 \pm 0.02$ \\
$\xi_{x}=0.4, \quad \xi_{y}=4.4$ & $2.59 \pm 0.07 \%$ & $1.75 \pm 0.02 \%$ & $0.96 \pm 0.02$ \\
$\xi_{x}=2.4, \quad \xi_{y}=4.4$ & $2.63 \pm 0.07 \%$ & $1.93 \pm 0.02 \%$ & $0.99 \pm 0.02$ \\
\hline
\end{tabular}

chromaticities of Table II.

\section{OFF-MOMENTUM TRANSVERSE DYNAMICS}

In this section we examine the mechanism of Touschek scattering in more detail. From radiation measurements we know that the highest radiation levels (and thus the highest loss areas) occur at the location in the ring where the vertical vacuum chamber size is the smallest. At this location, where the vertical beta function is $5 \mathrm{~m}$, the chamber height is $\pm 4 \mathrm{~mm}$. Therefore we can conclude that after a particle is Touschek scattered, it can reach large vertical amplitudes. It is also known from momentum aperture measurements that the dynamic aperture is limiting the Touschek lifetime. In fact these observations can be well understood by looking at the transverse particle dynamics: resonance excitation and orbit diffusion drive particles to such high vertical amplitudes as explained in this section.

\section{A. Off-momentum simulations}

The left side of Figure 5 shows a cartoon of the particle motion in the momentum and horizontal amplitude space for a particle scattered to an initial value of $\left(\delta_{0}, \sqrt{\beta_{x} H_{x}} \delta_{0}\right)$. The open circle indicates the initial coordinates of the particle. After the particle is scattered, there is a rapid oscillation in $\delta$ - so called synchrotron oscillations - and a slow damping in $\delta$ and horizontal amplitude back to the nominal orbit (the filled square in the figure). The time scales involved in these processes are very different - the synchrotron oscillation period is on the order of 100 turns whereas the damping time on the order of 10,000 turns. It should be noted that in this cartoon the damping times have been artificially increased by a factor 10 for illustration.

The right hand side of Figure 5 shows the tune change in the frequency space. The nominal (zero amplitude) tune values are $\nu_{x}=14.25$ and $\nu_{y}=8.20$ (the filled square in the figure). Just after being scattered, both the horizontal and vertical tunes are shifted to smaller values (the open circle in the figure) because of the tune shift with energy (chromaticities) and the tune shift with betatron amplitude. For the case of the ALS, the cross terms 

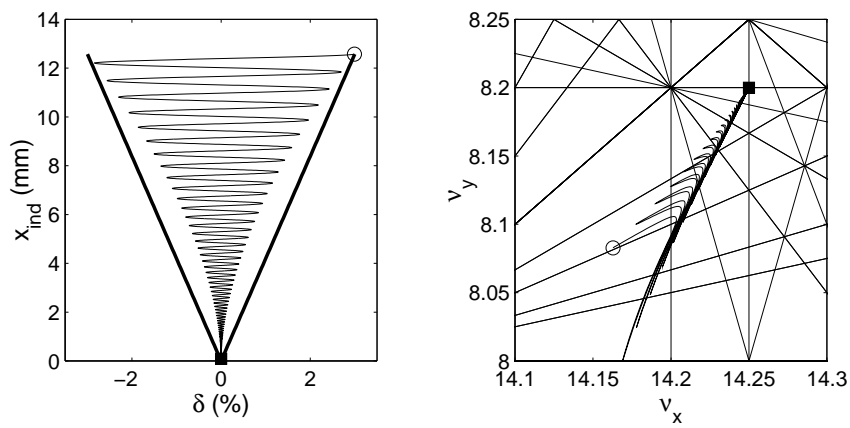

FIG. 5: Left: Schematic of particle behavior after Touschek scattering. Initial particle position after being scattered (open circle) then oscillating in energy and amplitude (solid line) and damping back down to the nominal orbit (filled square). Right: particle motion tracked in the tune space $\left(\nu_{x}, \nu_{y}\right)$, showing the effect of tune shift with betatron amplitude and tune shift with energy. Resonances up to the fifth order are shown.

of the tune shift with amplitude are largest. Therefore a large horizontal oscillation amplitude changes mostly the vertical tune.

Then as the particle undergoes momentum oscillations and damping, its tunes are slowly modulated back and forth in the transverse tune plane eventually returning to the nominal tune values. During this process it is possible that some of the particles may encounter a region in tune space where their motion is resonantly or diffusively excited to large vertical amplitudes where they may collide with the vacuum chamber.

An example of this is shown in Figure 6. We take the case of the machine adjusted to the nominal chromaticity, $\xi_{x}=0.4, \xi_{y}=1.4$. The linear machine model is fitted using measured response matrix data [24, 25] — linear coupling errors are also included. A particle is launched with initial conditions $x=12 \mathrm{~mm}, y=0.5 \mathrm{~mm}, \delta=2 \%$ and tracked using a full 6-dimensional tracking code including synchrotron oscillations and radiation damping [26-28]. The calculated horizontal, vertical, and longitudinal turn-by-turn positions are plotted (Figure 6) over 3,500 turns. In the vertical plane the vacuum chamber height is also drawn as dashed lines at $\pm 4 \mathrm{~mm}$. As seen in the figure the longitudinal oscillations appear stable and are slowly damping (due to radiation damping). However the amplitude of the horizontal and vertical oscillations are irregular and periods of rapid (chaotic) growth are observed. At some point (around turn 3,000) the particle reaches vertical amplitudes larger than $\pm 4 \mathrm{~mm}$. Therefore this particle is lost on the vacuum chamber.

Unfortunately it is difficult to exactly simulate this process experimentally. The reason is that in order to experimentally simulate Touschek scattering one would like to have an instrument (or set of instruments) that would simultaneously change the energy and amplitude of the beam within one turn. Although it is possible to give a single-turn large transverse kick to the beam, it is

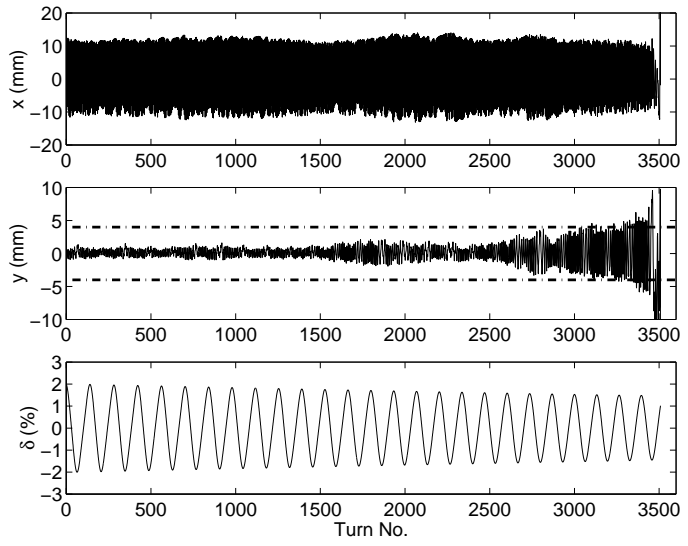

FIG. 6: Tracking with synchrotron oscillations and radiation damping for a particle launched with initial amplitude of $x=$ $12 \mathrm{~mm}, y=0.5 \mathrm{~mm}, \delta=2 \%$. At certain times the motion of the particle is highly diffusive causing the vertical oscillation amplitude to become larger than $\pm 4 \mathrm{~mm}$. In the ring, it would lead to particle loss on the vacuum chamber.

impossible to give a single-turn energy kick of a tenth of a $\mathrm{GeV}$ to a beam with an energy of $1.5 \mathrm{GeV}$. On the other hand it is possible to slowly (adiabatically) change the nominal energy of the beam. So instead of studying the full 6-dimensional case, we study the transverse dynamics at different momenta - the off-momentum transverse dynamics. We show in Section V that using this approach we gain a better insight into the details of the particle loss mechanisms.

Here we make the assumption that knowledge of the off-momentum dynamics is what is relevant to understand the full 6-dimensional dynamics. In doing so the transverse $\left(x, p_{x}, y, p_{y}\right)$ dynamics is viewed as being decoupled from the longitudinal $(\delta, l)$ dynamics. This decoupling is possible due to the large difference in time scales between the synchrotron oscillations (100 turns) and betatron oscillations (few per turn).

Figure 7 shows a cartoon of the method which we used to study the off-momentum transverse dynamics in the amplitude and frequency space. A particle is launched to a given horizontal amplitude then undergoes a damping in amplitude without executing any synchrotron oscillations (compare with Figure 5). This measurement can be used to identify amplitudes, momentum deviations, and frequencies where beam loss occurs - presumably by encountering regions where the motion is strongly nonlinear and chaotic. Therefore if the assumption that the beam loss is determined by the transverse dynamics is correct, this measurement is a way of locating the areas of beam loss that a Touschek scattered particle might experience.

\section{B. Off-momentum Frequency Map Analysis}

In a previous study [29] we showed that it was possible to experimentally measure resonant and chaotic behavior 

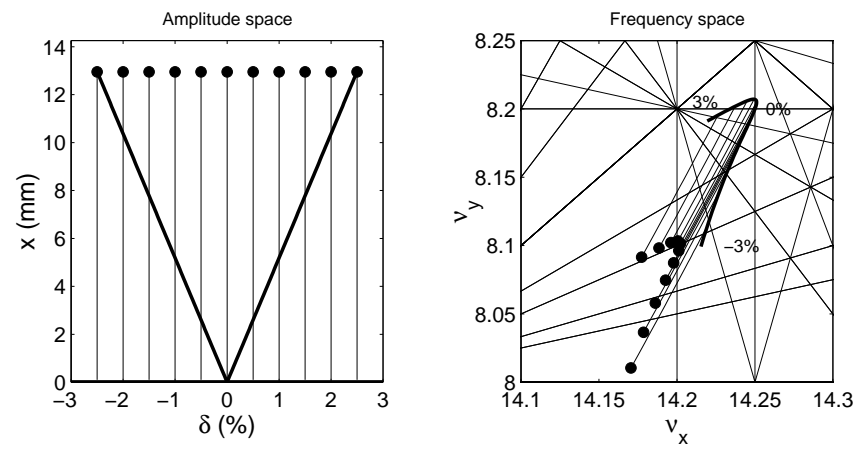

FIG. 7: Schematic of the measurement technique used to study the off-momentum dynamics. The particles starting at some horizontal oscillation amplitude (filled circles) damp down to the nominal orbit without any synchrotron oscillations for a fixed momentum deviation, $\delta$. Left: configuration space with induced amplitude after a Touschek scattering (diagonal lines). Right: tracked particle in tune space (curved line indicates tune shift with energy for particles near the closed orbit, diagonal lines indicate the tune shift with betatron amplitude).

in a storage ring using Frequency Map Analysis (FMA) [30-32]. It was also demonstrated that we could correlate particle loss to the zones with high diffusion [33]. In that study we were only looking at the on-momentum dynamics which is important for the understanding of particle loss during the injection process. In the present study we are extending and modifying these techniques to probe the off-momentum dynamics.

In Figure 8 FMA is used to explore the off-momentum dynamics. The machine model is the same as the one used to generate the tracking data in Figure 6. A frequency map is drawn at three different values of the relative momentum deviation, $\delta(-3 \%, 0 \%$, and $+3 \%)$. To generate each of the three maps the energy is fixed and particles are launched with various different initial horizontal and vertical amplitudes and tracked for $\mathrm{N}=1,000$ turns without synchrotron oscillation or damping. For surviving particles tunes are then computed with FMA and plotted in the frequency space. Because of the tune shift with energy, the maps of the three cases do not lie on top of each other. The dynamics encountered by the beam changes a lot with the energy: resonance orders and widths, diffusion rates, tune shifts with amplitude. As a result the stability area shrinks drastically for $\delta=-3 \%$.

The color scheme represents the orbit diffusion defined as $D=\frac{1}{N} \log _{10}\left\|\nu_{2}-\nu_{1}\right\|$ where $\nu_{1}$ and $\nu_{2}$ are the transverse tunes respectively determined over the first and last 500 turns of the particle tracking [31]. Blue regions describe stable dynamics and red and yellow regions stand for strong nonlinearity and chaos. As an example for the $\delta=0 \%$ map a large chaotic zone exists in the vicinity of the tunes of $\nu_{x}=14.2$ and $\nu_{y}=8.1$ (see Figure 8). This region is at the intersection of the third order resonance $\nu_{x}-2 \nu_{y}=-2$ and the fifth order resonance $5 \nu_{x}=71$. So a particle may be lost when its tunes are slowly sweeping

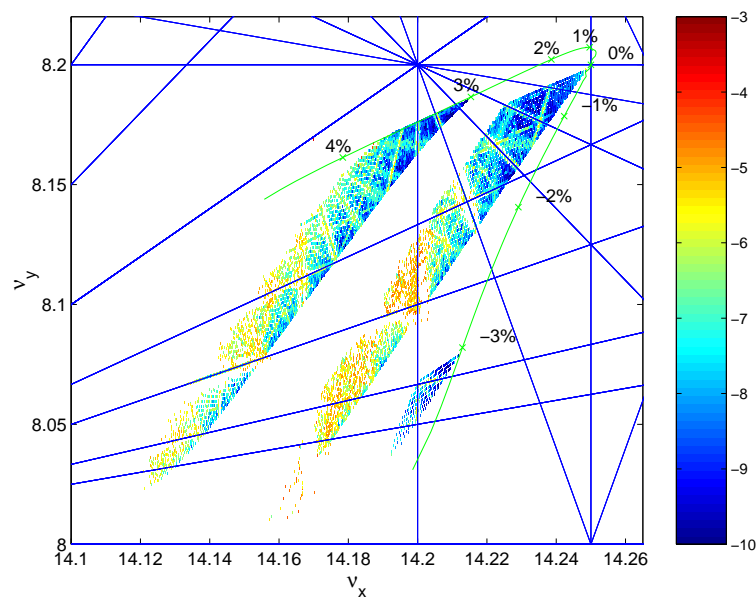

FIG. 8: Frequency maps for three different momentum deviations $(-3 \%, 0 \%$ and $+3 \%)$. The continuous green line indicates the tune shift with energy for particles near the closed orbit. The color code describes orbit stability from regular motion (blue) to chaotic motion (red). On top of the maps resonance lines are drawn up to the fifth order.

\section{over this region.}

To illustrate this we tracked a particle using the same model that we used to generate the frequency maps except that we now include synchrotron oscillations and radiation damping. A particle is launched with an initial condition corresponding to $x=10 \mathrm{~mm}, y=0.5 \mathrm{~mm}, \delta=$ 0\% (see Figure 9). The horizontal and vertical position is recorded each turn for 10,000 turns. Due to radiation damping the amplitudes tend to decrease, however at certain times (indicated by (c), (b) and (a) in the figure) there is rapid growth in the vertical plane.

One can see the corresponding position in the frequency space on the right side of Figure 9. The tunes were computed using FMA by dividing the 10,000 turnby-turn data into 40 blocks of 250 consecutive turns each. As observed in the figure, the particle is damping down to the nominal tunes. However, at certain points marked (c), (b) and (a), the particle trajectory diffuses to larger vertical amplitudes. The first change occurs at (c) when the trajectory crosses the $\nu_{x}-2 \nu_{y}=-2$ resonance. The second occurs at (b) when the trajectory crosses the $4 \nu_{x}+\nu_{y}=65$ resonance and there is a third region (a) where the particle crosses the resonance $3 \nu_{x}+2 \nu_{y}=59$. This behavior is consistent with the chaotic zones shown in the frequency map in Figure 8. For this particle the vertical amplitude only reaches a maximum of about $\pm 3 \mathrm{~mm}$ (near point (b)) - and thus this particle would not collide with the vacuum chamber (which is $\pm 4 \mathrm{~mm}$ ). However other particles with slightly different initial conditions can be excited to more than $\pm 4 \mathrm{~mm}$ vertically and will collide with the vacuum chamber. This suggests that the momentum aperture should be a strong function of the vertical physical aperture. Measurements detailing this are summarized in section VII. 

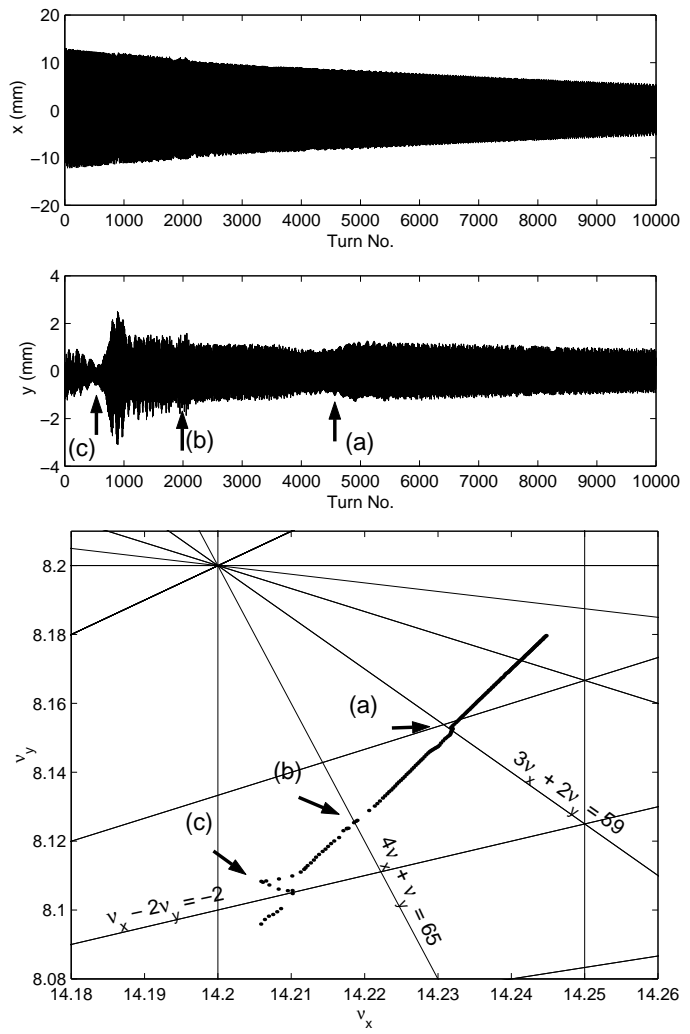

FIG. 9: Tracking of a particle with synchrotron oscillations and radiation damping (in tune and configuration space). When the trajectory crosses a region with high diffusion (see labels (a) to (c)), the vertical oscillation amplitude increases and at (c) the particle gets very close to the vacuum chamber of $4 \mathrm{~mm}$.

\section{EXPERIMENTAL MEASUREMENTS OF THE DYNAMIC APERTURE}

To study the off-momentum transverse dynamics at the ALS storage ring three tools have been used:

- The first tool is a set of two fast pulsed magnets called "pinger magnets": each pinger magnet's pulse duration is only $600 \mathrm{~ns}$. This is less than the time it takes for electrons to execute one turn around the ring. Therefore these magnets can provide a single-turn transverse kick to the electrons. The amplitudes of the horizontal and vertical fields can be adjusted independently.

- The second tool consists of turn-by-turn horizontal and vertical beam position monitors (BPMs). Each turn, the BPMs measure the transverse center of charge of the electron beam as it revolves around the ring. The BPMs can store up to 1,024 consecutive data points and are synchronized with the pinger magnet pulse. In this way it is possible to record the beam position of the first 1,024 turns after the beam is kicked by the pinger magnets.
- The third tool is a beam current monitor (DCCT). This monitor can record the current before and after the beam has been kicked allowing to measure the relative beam loss.

In the experiments the nominal energy of the machine is shifted adiabatically by changing the RFfrequency [13]:

$$
\delta=-\frac{1}{\alpha_{c}} \frac{\Delta f_{\mathrm{RF}}}{f_{\mathrm{RF}}}
$$

Then the beam is kicked with increasing horizontal kick strength (keeping the vertical kick strength at a small constant value). The relative change in beam current as well as the turn-by-turn data for each point are recorded. The measurements can then be repeated at different values of $\delta$. It is worth pointing out that this technique works well for the ALS but may not be suitable in rings with large circumferences where shifting the RFfrequency can also cause a large change in the damping partition numbers (see Appendix).

\section{A. Normal chromaticity, on-momentum}

An example of raw current measurements as a function of the horizontal kick amplitude is plotted in Figure 10 for a momentum offset of $\delta=0 \%$. The storage ring parameters have been the same as in the cases described in Section III, i.e. tunes at 14.25 and 8.20 , emittance ratio at about $7 \%$. For this example the chromaticity of the machine was adjusted to the ring's nominal chromaticity $\left(\xi_{x}=0.4, \xi_{y}=1.4\right)$. The top plot in the figure shows the measured beam current $I$ as a function of the kick amplitude after each horizontal kick. The relative loss-rate $\left(I_{n}-I_{n-1}\right) / I_{n-1}$, is plotted below. Here one can identify several step-like increases in the loss-rate at $7.8 \mathrm{~mm}$, $9.6 \mathrm{~mm}$, and $10.6 \mathrm{~mm}$ indicated by (a), (b), and (c) in the figure, and there is a total beam loss at $12.6 \mathrm{~mm}$ as indicated by $(\mathrm{d})$.

The frequency analysis of the turn-by-turn data for each kick amplitude will show us the origins of these beam losses. Figure 11 displays the measured frequencies $\left(\nu_{x}, \nu_{y}\right)$. In this plot the size of the dot is related to the relative loss-rate $\left(\left(I_{n}-I_{n-1}\right) / I_{n-1}\right)-$ for legibility zero loss corresponds to a finite dot size. First at a zero kick amplitude tunes correspond to $\nu_{x}=14.25$ and $\nu_{y}=8.20$ (indicated by a (o) in the figure). As the kick amplitude increases the tunes shift to smaller values. Around $\left(\nu_{x}, \nu_{y}\right)=(14.23,8.15)$ (see point (a) in Figure 10), a slight beam loss is observed associated to the crossing of the resonance $3 \nu_{x}+2 \nu_{y}=59$. The neighborhood of this resonance, reached at a $7.8 \mathrm{~mm}$ kick amplitude, can be identified as the lowest amplitude region harmful for the dynamics. Then at tunes of $\nu_{x}=14.22$ and $\nu_{y}=8.11$ there is a more significant change in the loss rate (b). This occurs as the tune crosses the 5th order resonance 

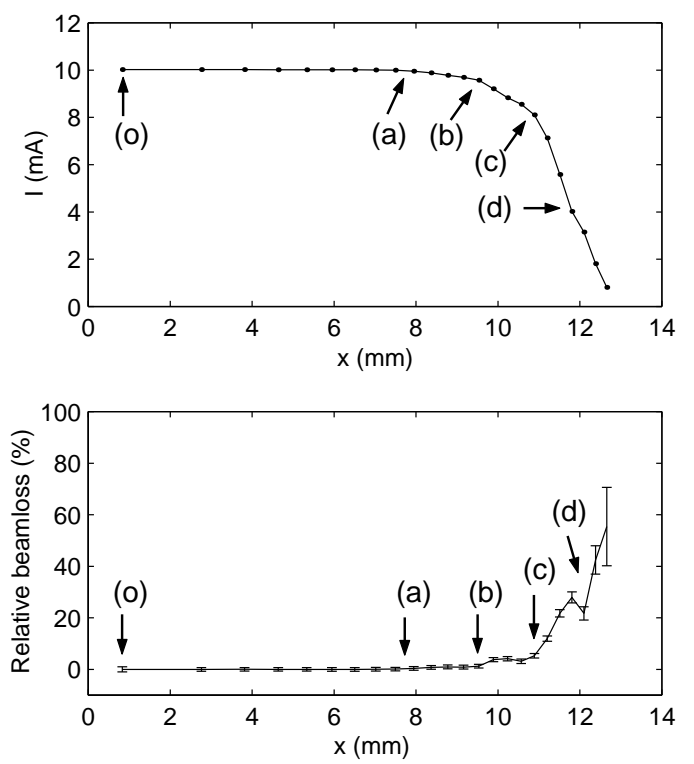

FIG. 10: Upper: Beam current versus kick amplitude. Lower: First derivative of beam current versus kick amplitude. Each change in beam loss is labeled by a letter.

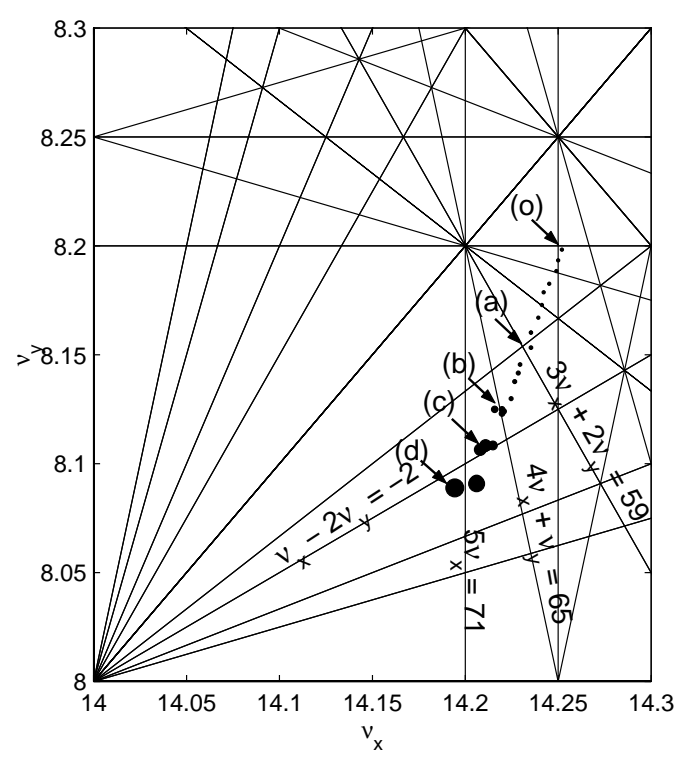

FIG. 11: Tunes and loss-rates as calculated from turn-byturn BPM data and DCCT measurements. The labels defined as in Figure 10 indicate beam loss (dot size) increase near particular resonance lines.

$4 \nu_{x}+\nu_{y}=65$. The loss rate further increases as the tune crosses the third order resonance $\nu_{x}-2 \nu_{y}=-2$ as indicated by a (c) in Figure 11 and Figure 10 at an amplitude of $10.6 \mathrm{~mm}$. And finally there is a total beam loss on the fifth order resonance $5 \nu_{x}=71$ as indicated by (d). Thus using the frequency data enables us to identify which resonances are responsible for particle loss.

A comparison of the experimental data (Figures 10 and 11) with the theoretical simulation (Figures 8 and 9) reveals a consistent picture of the dynamics. The tune shifts with amplitude are the same and the high diffusion zones in the simulation correspond to regions of significant particle loss in the experiment.

Up to now only data for a single value of $\delta$ have been shown. We will now present data for various momentum offsets and 3 different values for the chromaticities.

\section{B. Normal chromaticity, off-momentum}

Figure 12 shows the results for the nominal chromaticities $\left(\xi_{x}=0.4, \xi_{y}=1.4\right)$. The relative loss-rates are plotted both in the amplitude space $(x, \delta)$ (left) and in the frequency space (right). In the amplitude space, the induced amplitude versus energy of a particle that has been Touschek scattered in the arcs is indicated by a red dashed line; in the frequency space the change of tune versus energy at zero amplitude is indicated by a solid green line.

As shown in the previous section it is possible to identify resonances and chaotic regions that limit the apertures by looking at the data plotted in the frequency space. In this case several dangerous resonances or intersections of resonances responsible for beam loss can be identified. To help distinguish between different momentum offsets we have plotted points with negative momentum deviation in blue, on-momentum points in black, and positive momentum deviation points in red. For large negative momentum deviations the particle loss at high amplitudes is caused by the integer resonance $\nu_{y}=8$ (area A in Figure 12). Around zero momentum deviation the particles are lost in the neighborhood of several resonances $\left(5 \nu_{x}=71, \nu_{x}-2 \nu_{y}=-2\right.$, see area $\left.\mathrm{B}\right)$. At large positive momentum deviations the loss is again due to the integer resonance $\nu_{y}=8$ (area $\mathrm{C}$ ) and the linear coupling resonance $\nu_{x}-\nu_{y}=6($ area $\mathrm{D})$.

Now that the amplitude and cause of the beam loss have been found, it is possible to relate this case to the fully 6-dimensional case including synchrotron oscillations. In this way we can determine the limitation of the dynamic and momentum aperture. When a particle is Touschek scattered we have previously seen that it oscillates rapidly in momentum and its amplitude damps slowly in momentum and transverse amplitudes towards the beam center. Figure 5 shows the shape of the footprint of the particle trajectory in configuration space. The footprint looks similar to an inverted triangle. In order to find the largest momentum deviation a particle can encounter without being lost, we have to find the largest inverted triangle which misses all high-loss areas: As one can see in Figure 12, a particle that has been Touschek scattered in the arcs up to $|\delta| \leq 2.5 \%$, corresponding to an induced horizontal amplitude of $\pm 9 \mathrm{~mm}$ will cross no region of large loss. However there are large loss regions at larger energy deviations and amplitudes - the first being at $10 \mathrm{~mm}$ (both at $\delta=-2.5 \%$ and at $\delta=0.5 \%$ ). From this measurement we can infer that the 

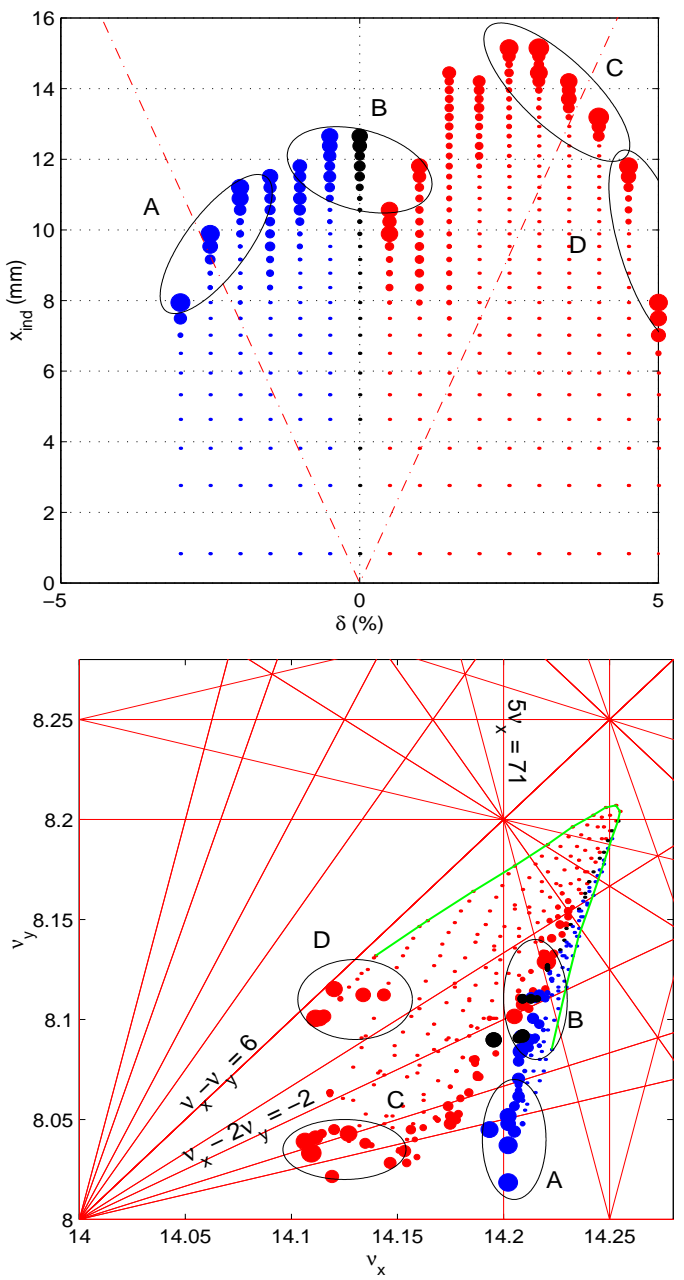

FIG. 12: Measured momentum aperture in tune (bottom) and in configuration space (top) for the chromaticity set $\left(\xi_{x}=\right.$ $\left.0.4, \xi_{y}=1.4\right)$. Point size indicates relative beam loss and labels point out specific resonance areas responsible for these losses. Resonances up to the fifth order are drawn in the tune space.

limit of the dynamic momentum aperture in the arcs is slightly more than $2.5 \%$. This compares well with the value of $2.65 \%$ (see Table II) measured using the technique described in Section III.

Through this measurement, the global dynamics of the storage ring is revealed. Not only are the amplitude of loss regions and the size of the dynamic aperture determined, but also the resonances that are responsible for loss are identified. All this provides us with a comprehensive understanding of the beam dynamics for this particular machine condition.

\section{Large vertical chromaticity, off-momentum}

Figure 13 shows the results for the second case with the higher vertical chromaticity $\left(\xi_{x}=0.4, \xi_{y}=4.4\right)$. When comparing with the nominal chromaticity case shown in Figure 12, we see that the aperture is reduced in several areas. In particular we wish to point out the reduction in the aperture at $-2 \%$ and the large loss region extending from $1.5 \%$ to $5 \%$. In fact this loss region at positive $\delta$ causes the lowest limit of the momentum aperture in the arcs. It limits the aperture at a momentum deviation of slightly more than $\delta=1.5 \%$, corresponding to an induced amplitude, $x_{\text {ind }}$, of slightly less than $7 \mathrm{~mm}$. The second limitation at negative $\delta$ limits the aperture to less than $\delta=-2.0 \%$ (corresponding to an induced amplitude of slightly more than $7 \mathrm{~mm}$ ).
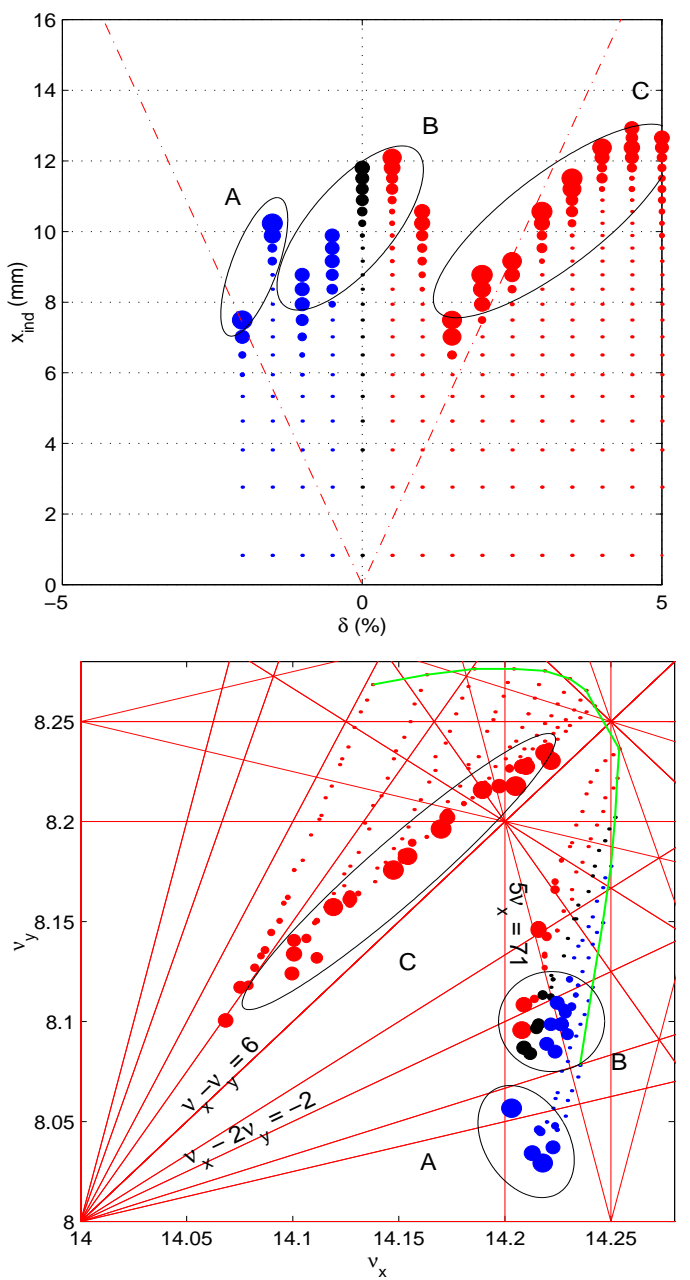

FIG. 13: Measured momentum aperture in tune (bottom) and in configuration space (top) for the chromaticity set $\left(\xi_{x}=\right.$ $\left.0.4, \xi_{y}=4.4\right)$. Point size indicates relative beam loss and labels point out specific resonance areas responsible for these losses. Resonances up to the fifth order are drawn in the tune space.

By looking at the frequency plot we see that the plot is very different from the nominal chromaticity case. The negative $\delta$ limit corresponds to the integer resonance $\left(\nu_{y}=8\right)$. This is true as well for the normal chromaticity case but due to the larger vertical chromaticity, this limit 
is reached at lower values of $|\delta|$. Note a second loss region (B) similar to loss region (B) in Figure 12. The lowest limitation occurs when the particle frequency approaches the linear coupling resonance - a resonance that is deliberately excited to control the vertical beam size. The reason that the coupling resonance is important is that the tune shift with energy in the case with a larger vertical chromaticity moves the tune to the other side of the coupling resonance. Then the tune shift with amplitude moves the tune down onto the coupling resonance where there is beam loss. Based on this measurement one would predict that the dynamic aperture would restrict the momentum aperture in the arcs to slightly more than $1.5 \%$. This compares well with the value of $1.75 \%$ measured before (see Table II).

\section{Large horizontal and vertical chromaticity, off-momentum}

When looking at the results of the initial momentum aperture measurements in Section III it was not obvious why increasing the horizontal chromaticity by two units from $\xi_{x}=0.4$ to $\xi_{x}=2.4$ actually increased the momentum aperture. As previously stated this may seem counter intuitive, but by looking at the data from the dynamic aperture measurement, the reason becomes clear.

In the previous case with high vertical chromaticity, the linear coupling resonance limited the momentum aperture. So one way to enlarge the aperture is to increase the value of $\delta$ at which the tune crosses over the linear coupling resonance. This is precisely what happens when the horizontal chromaticity is increased. Figure 14 shows the results for the high horizontal and vertical chromaticities $\left(\xi_{x}=2.4, \xi_{y}=4.4\right)$. By increasing the horizontal chromaticity we shift the effect of the coupling resonance to larger positive momentum deviations, thus increasing the size of the aperture to about $2 \%$; now the lifetime is limited by the integer resonance. This compares well with the value of $1.9 \%$ measured before (see Table II).

It should be mentioned that we first studied the case of $\left(\xi_{x}=0.4, \xi_{y}=4.4\right)$, discovered the limitation caused by the coupling resonance, and then predicted that increasing the horizontal chromaticity would increase the lifetime. So this technique was used in a predictive way to improve the performance of the machine.

\section{SIMULATIONS OF THE DYNAMIC APERTURE}

The method presented in the previous section provides a model independent way to obtain a global picture of the off-momentum dynamics. It can be used to understand limitations of the dynamic momentum aperture and provide guidance on how to improve the machine performance. Furthermore it has been demonstrated that the
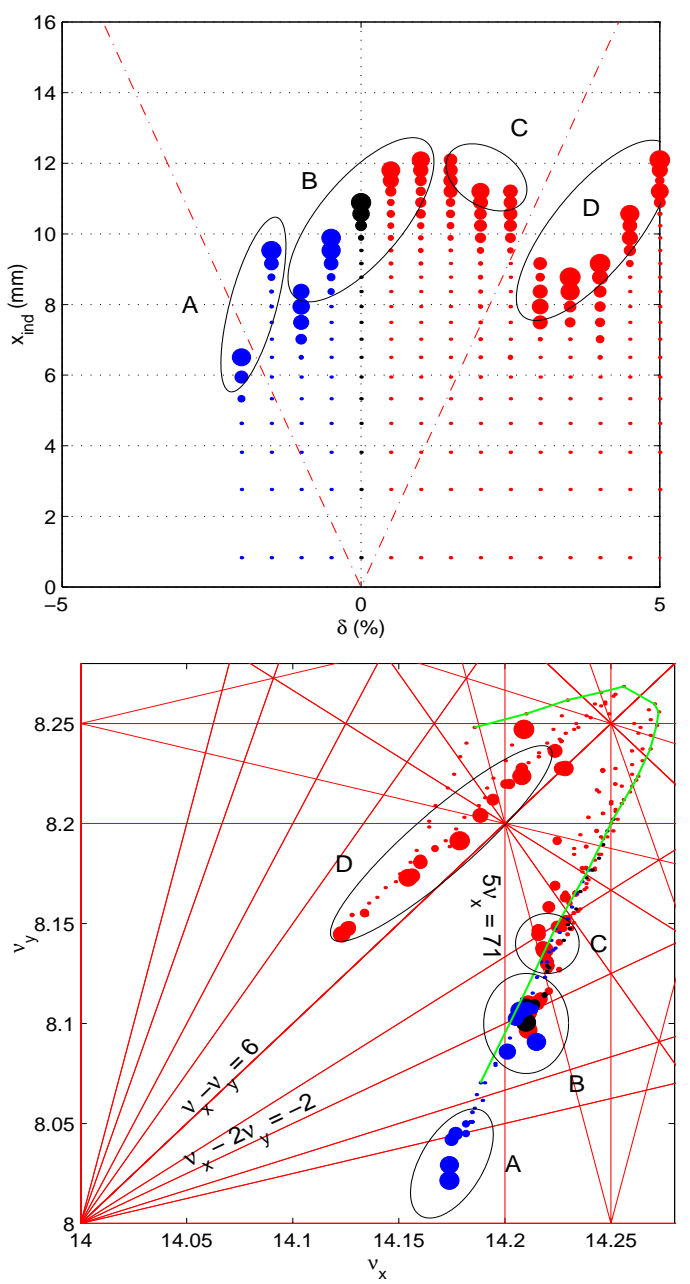

FIG. 14: Measured momentum aperture in tune (bottom) and in configuration space (top) for the chromaticity set $\left(\xi_{x}=\right.$ 2.4, $\left.\xi_{y}=4.4\right)$. Point size indicates relative beam loss and labels point out specific resonance areas responsible for these losses. Resonances up to the fifth order are drawn in the tune space.

mechanisms causing particle loss are well understood and that tracking simulations for individual particle trajectories agree well with the measured dynamics.

In order to evaluate, whether simulations reproduce the differences in momentum aperture found in the experiment, additional tracking studies were performed. Particle trajectories were tracked for a large set of initial conditions $(100 \times 100)$ in the configuration space formed by horizontal oscillation amplitude and momentum deviation. Afterwards, the transverse oscillation frequencies for each of those trajectories were calculated, similar to the method used in the experiment. We will concentrate here on the main difference of the two cases with high vertical chromaticity as an example. A full analysis will be the topic of future studies.

Figures 15 and 16 show the results of those simulations (tracking the particle coordinates for 1,000 turns without 
synchrotron radiation nor radiation damping) for the two cases with high vertical chromaticity. The color code in the plots again indicates the diffusion rate on a logarithmic scale (as in Figure 8). In addition, the measured normalized beam loss rates as a function of momentum deviation and initial horizontal oscillation amplitudes are shown as red dots in the plots (compare Figure 15 with Figure 13 and Figure 16 with Figure 14).

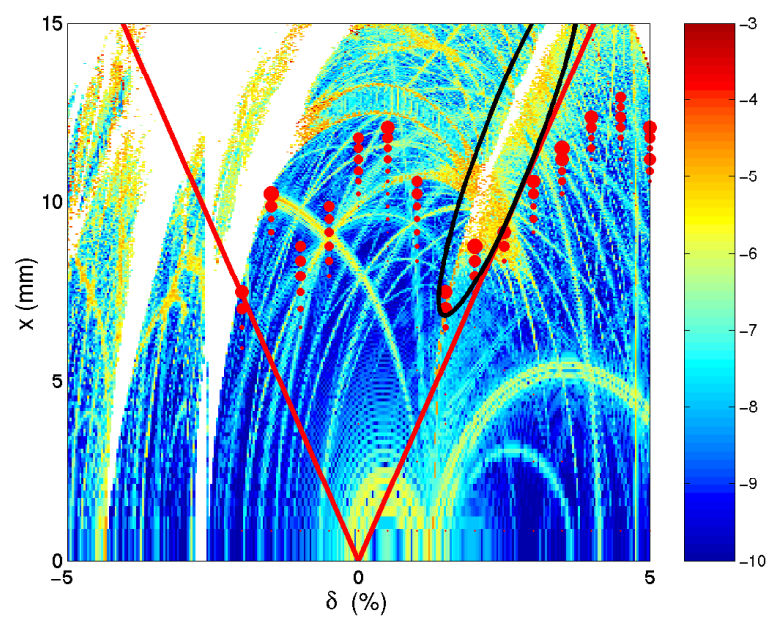

FIG. 15: Comparison of simulation of the dynamic momentum aperture and measurement of normalized beam loss in configuration space $\left(\xi_{x}=0.4, \xi_{y}=4.4\right)$. White areas indicate regions where the particles were lost during the tracking. The loss area caused by the coupling resonance $\nu_{x}-\nu_{y}=6$ is circled.

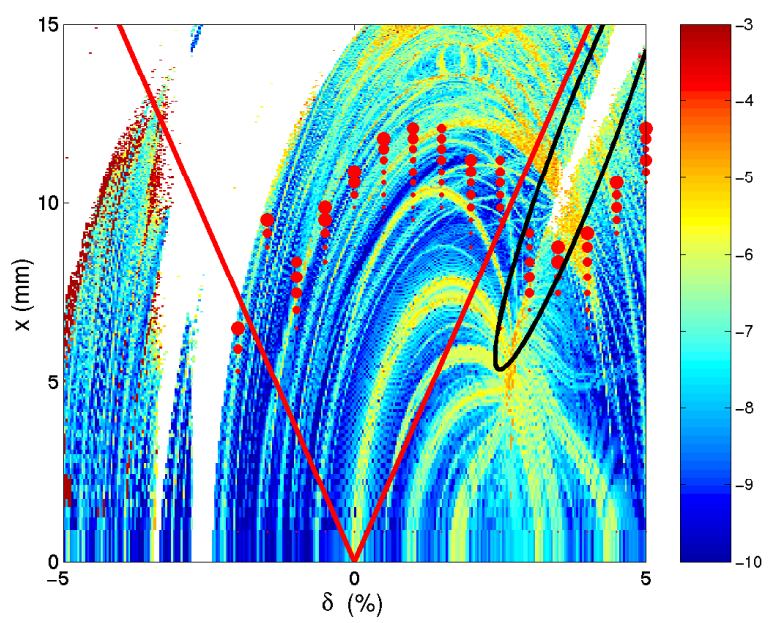

FIG. 16: Comparison of simulation of the dynamic momentum aperture and measurement of normalized beam loss in configuration space $\left(\xi_{x}=2.4, \xi_{y}=4.4\right)$. The loss area caused by the coupling resonance $\nu_{x}-\nu_{y}=6$ is circled.

The general features of the simulation and the measured beam loss agree well. Areas where particles were lost before reaching 1,000 turns in the simulation are clearly outside the stable areas in the measurements as well. In addition, in many cases one can associate an area with high diffusion in the simulation to areas with beam loss in the measurements. Comparing Figure 15 and Figure 16 one can see that the main difference due to the increased horizontal chromaticity in the measured data - the shift of the loss area caused by the coupling resonance, $\nu_{x}-\nu_{y}=6$ (circled area in the plots), to larger positive momentum deviations - is reproduced well in the simulations.

However, the figures show significantly more diffusive areas and more details than the measurements. Therefore it is not trivial to evaluate which of the diffusive areas might be harmful by just looking at this kind of plots. The main reason is that the particle loss happens in the vertical plane (as shown earlier), whereas in these figures, the configuration space of momentum deviation and horizontal oscillation amplitude is plotted. One example, where an area of high diffusion in these plots might not indicate a high-loss area, is if the diffusion happens in the horizontal amplitude configuration space (i.e. the particle trajectory remains at low vertical oscillation amplitudes).

Fortunately, there are several methods to figure out which diffusive areas can really cause beam loss. The first is to plot the simulation data in the tune space instead of configuration space (similar to the way it is done in the experimental case, compare Figures 12-14). That method can also help to identify which specific resonances are harmful and directly provide guidance how to improve the stability of trajectories. The second is to compare the simulation results with results of measurements (like it is done in this section). This allows to make very good predictions for situations not too different from the one which one made the measurements in. The last method employs the use of many simulated frequency maps (calculated in the configuration space formed by horizontal and vertical oscillation amplitude) for various, fixed momentum offsets (compare Figure 8). This way one can evaluate the possible directions of the diffusion in the full transverse phase space for a given momentum offset.

These simulation methods have been used successfully during the Superbend project at the ALS, where three normal conducting bending magnets in the middle of three of the twelve triple bend achromat cells have been replaced by superconducting ones [34]. This installation broke the original 12-fold symmetry of the ALS and therfore changed the nonlinear single particle dynamics significantly. The predictions of the simulation proved to be quite accurate. Nevertheless, it should be mentioned that the results shown in Figures 15-16 depend very much on the initial phases. Therefore, these studies should be continued and completed by an equivalent analysis in the frequency domain, where the results will be more independent of the phases [35]. 


\section{DEPENDENCE OF THE MOMENTUM APERTURE ON THE VERTICAL PHYSICAL APERTURE}

The loss of particles due to resonant excitation of their motion or due to diffusion happens mostly in the vertical plane (compare Section IV). Therefore one should expect that the momentum aperture depends on the vertical aperture. To study this effect quantitatively, measurements of the dependence of the momentum aperture of the ALS on the vertical physical aperture were also performed.

In the measurements, the vertical physical aperture was set to various values by using a vertical scraper. The scraper-like the narrow gap vacuum chambers which normally restrict the vertical physical aperture to about $\pm 4 \mathrm{~mm}$ - is located in one of the twelve straight sections of the ALS where the vertical beta function is about $4 \mathrm{~m}$. For a given scraper setting the beam lifetime was measured as a function of the RF-momentum aperture, $\varepsilon_{R F}$, and the momentum apertures, $\varepsilon_{\text {straight }}$ and $\varepsilon_{\text {arc }}$, were determined from a fit to this data using Equation 9, similar to what was done to generate Figure 3. The results of the measurement are shown in Figure 17. One can see that the (transverse) momentum aperture in the arcs is a strong function of the vertical physical aperture. It decreases monotonously for physical aperture smaller than the nominal $\pm 4 \mathrm{~mm}$ and becomes nearly zero around a vertical physical aperture of about $1 \mathrm{~mm}$. One should remark that the vertical beam size at the location of the scraper for typical emittance coupling is $\sigma_{y} \leq 30 \mu \mathrm{m}$, which means that $\pm 1 \mathrm{~mm}$ still corresponds to a physical aperture of more than $\pm 30 \sigma_{y}$.

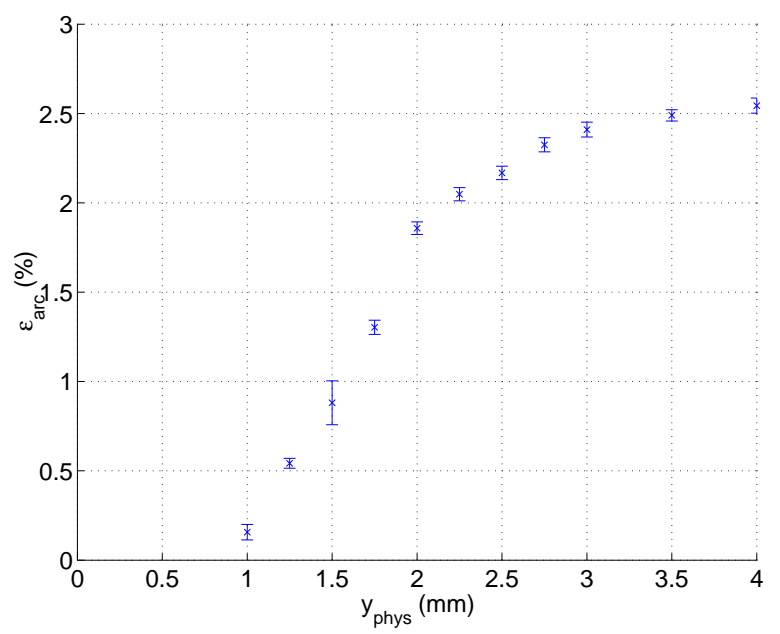

FIG. 17: Measurement of the momentum aperture in the arc, $\varepsilon_{\text {arc }}$, as a function of the vertical physical aperture $y_{\text {phys }} \cdot \varepsilon_{\text {arc }}$ was calculated by individually fitting the results of lifetime measurements as a function of the RF-momentum aperture for various settings of a vertical scraper. $\varepsilon_{\text {straight }}$ was larger than $3 \%$ for all scraper settings.

Another result of the measurement was that the mo- mentum aperture in the straight sections did remain at values larger than $3 \%$ (i.e. at values larger than the RF-momentum aperture achievable with the current RFsystem of the ALS) for all settings of the scraper all the way down to a vertical physical aperture of only $1 \mathrm{~mm}$. Based on the understanding of the loss mechanism as described in Section IV this was the expected behavior.

The fact that the momentum aperture and therefore the beam lifetime is a strong function of the vertical physical aperture is of special importance for synchrotron light sources. Since the performance (photon brightness) which can be achieved with an undulator depends critically on the gap of the magnetic undulator structure, there is a strong incentive to make the undulator gap and therefore the physical aperture of the vacuum chamber as small as possible. Recently several second and third generation synchrotron light sources have been equipped with insertion devices with smaller and smaller gaps. The measurements presented here underline that it is very important to study the impact which the reduced vertical physical aperture has on the momentum aperture in addition to studies of vacuum, impedance and radiation issues.

\section{CONCLUSION}

Short beam lifetimes are one of the major performance limitations in particle storage rings. The beam lifetime in many particle storage rings is limited by particles being Touschek scattered outside of the ring's momentum aperture. Up to now the beam lifetimes which have been realized have been smaller than predicted as a result of the momentum aperture limitations. Measurements at the ALS as well as other storage rings have demonstrated that the dynamic aperture is the dominant effect determining the size of the momentum aperture.

Employing the method of frequency analysis to study the off-momentum transverse dynamics provides a very powerful model independent diagnostic tool to visualize the global dynamics of the system and understand the aperture limitations. In particular, this tool helps to identify regions in the frequency space where beam loss occurs. The present study demonstrates that the knowledge of the off-momentum transverse dynamics allows accurate prediction of the momentum apertures at the ALS and can suggest strategies to improve its dynamics. Even with just two sextupole families at the ALS, the knowledge of the off-momentum dynamics allowed us to adjust the chromaticity (increasing the horizontal chromaticity) resulting in increased lifetimes by $25 \%$.

The agreement is very good between the estimated dynamic momentum aperture from the measurements using a pinger magnet and the direct measurement using the RF-amplitude scans. As mentioned in Section IV, since it is not possible to give a single-turn momentum kick to the beam with a large amplitude, it was not possible to simulate exactly the full 6-dimensional dynamics of Tou- 
schek scattering. It was only possible to study the offmomentum dynamics. The results presented here are a demonstration of the validity of the assumption (made in Section IV) that the knowledge of the off-momentum dynamics is sufficient to understand the full 6-dimensional dynamics.

However, even if it would be possible to study the full 6-dimensional dynamics, the time span limits imposed by decoherence would not allow us to separate the synchrotron oscillation contributions to the betatron frequencies. So there is an additional advantage of studying the off-momentum dynamics for fixed momentum offsets: Using the beam oscillation data, which was recorded as additional information, one can identify the resonances in tune space that cause those loss regions. The analysis showed that the large difference in lifetime is not caused by a large difference in strength of resonances, but by the fact that different linear chromaticities cause the particles to sample different regions in phase space, and thus experience different sets of resonances (Section V).

The agreement between measured data and simulation results which are based on calibrated machine models is good. The simulations show that the loss regions correspond to resonances and intersections of resonances where the particle motion is resonantly or diffusively excited to large vertical amplitudes, causing the particle to collide with the vacuum chamber. This is consistent with the fact that the highest loss rates are measured at the narrowest vertical gap chambers. These studies provide both confidence in the model and the possibility to accurately predict the performance of upgrades to the ALS.

In most other storage rings, knowledge of the offmomentum dynamics will allow to accurately predict the momentum aperture. Therefore these techniques will be important for understanding and improving the momentum apertures in existing storage rings as well as precisely predicting the momentum apertures in future accelerators.

\section{Acknowledgments}

The authors would like to thank the staff at the ALS, particularly Charles Kim and Hiroshi Nishimura with whom we had many interesting discussions, and Ben Feinberg for helpful comments on this paper. We wish to thank Louis Emery, Kathy Harkay, Vadim Sajaev and Kwang Je Kim at the Advanced Photon Source for interesting discussions and pointing out the difficulty of studying the off momentum dynamics in large storage rings.

This work was supported by the Director, Office of Energy Research, Office of Basic Energy Sciences, Materials Sciences Division of the U.S. Department of Energy, under Contract No. DE-AC03-76F00098

\section{APPENDIX: DAMPING PARTITION NUMBERS}

The radiation of charged particles circulating in a storage ring causes a slow damping of the betatron and syn- chrotron oscillation amplitudes of the particle. In the study of the off momentum dynamics, the reference momentum is adjusted by changing the RF frequency. However this also modifies the distribution of damping between the different planes. Because of this the motion can become antidamped in one plane making the technique presented in this paper unsuitable. This is particularly important for large rings.

The damping times $\left(\tau_{x}, \tau_{y}, \tau_{z}\right)$ are inversely proportional to the damping partition numbers $\left(J_{x}, J_{y}, J_{z}\right)$ whose values are, for a storage ring with mid-plane symmetry [20]:

$$
J_{x}=1-D, \quad J_{y}=1, \quad J_{z}=2+D,
$$

with

$$
D=\frac{\oint \frac{\eta}{\rho}\left(\frac{1}{\rho^{2}}+2 K\right) d s}{\oint \frac{1}{\rho^{2}} d s}
$$

where $\rho, K$ and $\eta$ are respectively the local curvature radius, the linear field gradient, and the dispersion function of a magnet. The motion of the particles is simultaneously damped in the horizontal and longitudinal planes $\left(J_{x, z}>0\right)$ if $D$ satisfies the stability condition:

$$
-2<D<1
$$

When varying the RF-frequency, the closed orbit shifts in the quadrupoles which are located in regions of finite dispersion $\left(\eta_{Q}\right)$. Therefore the value of $D$ (to first order) changes by:

$$
\begin{aligned}
\Delta D & \approx 2 \frac{\rho_{D}^{2}}{N_{D} L_{D}} \delta \sum_{\text {QuadFamilies }} N_{Q} L_{Q}\left(K_{Q} \eta_{Q}\right)^{2} \\
& =A \times \delta
\end{aligned}
$$

where $N_{Q}, L_{Q}$, and $K_{Q}$ are respectively the number, the length and gradient for each family of quadrupoles (dipoles). Stating $D=D_{0}+\Delta D$ and combining Equation A.3 and A.4 the stability condition becomes:

$$
\frac{-2-D_{0}}{A}<\delta<\frac{1-D_{0}}{A}
$$

For the ALS the full range in which there is damping in both planes is $\Delta \delta=3 / A$ which is $33 \%(-18 \%,+16 \%)$. This is much larger than the range of interest for the study of the off-momentum dynamics $( \pm 5 \%)$.

However for larger rings this may not be the case anymore. A small change in momentum gives a large change in damping rates. For instance, at the Advanced Photon Source in Chicago [36], the range is $-2.8 \%<\delta<$ $1.4 \%$ [37]. This limits this technique's usefulness in exploring the off-momentum transverse dynamics in larger rings. 
[1] 1-2 GeV Synchrotron Radiation Source Conceptual Design Report, LBNL publication PUB-5172, Rev. (1986).

[2] PEP-II Conceptual Design Report, LBL-PUB-5379 (1993).

[3] The ESRF Red Book, Grenoble (1987).

[4] W. Decking, J. Byrd, C. Kim, and D. Robin, in Proceedings of the 6th European Particle Accelerator Conference, Stockholm, 1998 (Institute of Physics, Bristol, UK, 1998), 1262-1264.

[5] W. Decking, and D. Robin, in Proceedings of the 6th European Particle Accelerator Conference, Stockholm, 1998 (Institute of Physics, Bristol, UK, 1998), 1265-1267.

[6] W. Decking, and D. Robin, in Proceedings of the AIP Conference 468, Arcidosso, Italy, 1998 (Woodbury, New York, 1999), 119-128.

[7] A. Ropert, in Proceedings of the 6th European Particle Accelerator Conference, Stockholm, 1998 (Institute of Physics, Bristol, UK, 1998), 629-631.

[8] A. Ropert, in Proceedings of the 18th Particle Accelerator Conference, New York, 1999 (IEEE, Piscataway, NJ, 1999), 2328-2330.

[9] A. Ropert, J.M. Filhol, P. Elleaume, L. Farvacque, L. Hardy, J. Jacob, and U. Weinrich, in Proceedings of the 7th European Particle Accelerator Conference, Vienna, 2000 (Austrian Academy of Sciences Press, Vienna, 2000), 83-87.

[10] M. Takao, K. Soutome, and H. Tanaka, in Proceedings of the 7th European Particle Accelerator Conference, Vienna, 2000 (Austrian Academy of Sciences Press, Vienna, 2000), 1569-1571.

[11] U. Wienands, in Proceedings of the 19th Particle Accelerator Conference, Chicago (IEEE, Piscataway, NJ, 2001), $2758-2760$

[12] J. LeDuff, Nucl. Instr. \& Methods, A239, 83 (1985).

[13] H. Bruck, Accélérateurs Circulaires de Particules, (Presses Universitaires de France, Paris, 1966).

[14] F. Porter, Nucl. Instr. \& Methods, A302, 209 (1991)

[15] J.L. Laclare, in Proceedings of the 6th European Particle Accelerator Conference, Stockholm, 1998 (Institute of Physics, Bristol, UK, 1998), 78-82.

[16] J. Bengtsson, W. Joho, P. Marchand, G. Muelhaupt, L. Rivkin, and A. Streun, Nucl. Instr. \& Methods, A404, 237-247 (1998).

[17] M.P. Level et al., in Proceedings of the 6th European Particle Accelerator Conference, Stockholm, 1998 (Institute of Physics, Bristol, UK, 1998), 599-601.

[18] M. Boege, and A. Streun, in Proceedings of the 18th Particle Accelerator Conference, New York, 1999 (IEEE, Pis- cataway, NJ, 1999), 2430-2432.

[19] W. Decking, and D. Robin, in Proceedings of the 18th Particle Accelerator Conference, New York, 1999 (IEEE, Piscataway, NJ, 1999), 1580-1583.

[20] M. Sands, The Physics of Electron Storage Rings: an Introduction, University of California, SLAC-121 (1970).

[21] Y. Wu., V. N. Litvinenko, B. Burnham, S. H. Park, and J. M. J. Madey, IEEE Transactions on Nuclear Science, 44, 5, 1753-1759 (1997).

[22] E.D. Courant, and H.S. Snyder, Ann. Phys., 3, 1-48 (1958).

[23] A. Nadji, G. Flynn, J.L. Laclare, M.P. Level,P. Nghiem, and J. Payet, in Proceedings of the 17th Particle Accelerator Conference, Vancouver, 1997 (IEEE, Piscataway, NJ, 1997), 1517-1519.

[24] J. Safranek, Nucl. Instr \& Methods, A388, 27 (1997)

[25] D. Robin, J. Safranek, and W. Decking, Phys. Rev. ST Accel. Beams 2, 044001 (1999).

[26] R. Ruth, IEEE Trans. Nucl. Sci., 30, 2669-2671 (1983).

[27] E. Forest, Beam Dynamics: a new Attitude and Framework (Harwood Academic Publishers, Chur, Switzerland, 1998).

[28] A. Terebilo, in Proceedings of the 19th Particle Accelerator Conference, Chicago (IEEE, Piscataway, NJ, 2001), 1692-1694.

[29] D. Robin, C. Steier, J. Laskar, and L. Nadolski, Phys. Rev. Lett., 85, 3, 558 (2000).

[30] J. Laskar, Icarus, 88, 266-291 (1990).

[31] H.S. Dumas, and J. Laskar, Phys. Rev. Lett., 70, 29752979 (1993).

[32] J. Laskar, in Proceedings of 3DHAM95 NATO Advanced Study Institutes, S'Agaro, 1995 (Kluwer Academic Publishers, Dordrecht, The Netherlands, 1999), 134-150.

[33] C. Steier,D. Robin, J. Laskar, and L. Nadolski, in Proceedings of the 7th European Particle Accelerator Conference, Vienna, 2000 (Austrian Academy of Sciences Press, Vienna, 2000), 1077-1079.

[34] D. Robin et al., in Proceedings of the 19th Particle Accelerator Conference, Chicago (IEEE, Piscataway, NJ, 2001), 2632-2634.

[35] J. Laskar, Physica D, 67, 257-281 (1993)

[36] 7-GeV Advanced Photon Source Conceptual Design Report, Argonne National Laboratory, ANL-87-15 (1987).

[37] J. Murphy, Synchrotron Light Source Data Book, Brookhaven National Laboratory, Report BNL-42333 (1996). 